\title{
Effects of radiofrequency on adipose tissue: A systematic review with meta-analysis
}

\author{
Ana Luísa Vale ${ }^{1}$ Graduation in Physiotherapy | Ana Sofia Pereira ${ }^{1}$ Graduation in \\ Physiotherapy | Andreia Morais ${ }^{1}$ Graduation in Physiotherapy | Andreia Noites ${ }^{3}$ PhD | \\ Adriana Clemente Mendonça ${ }^{2}$ PhD | Juliana Martins Pinto ${ }^{2}$ PhD | Rui Vilarinho ${ }^{3}$ MSc | \\ Paulo Carvalho ${ }^{3} \mathrm{PhD}$ \\ ${ }^{1}$ School of Allied Health Technologies, ${ }^{2}$ Department of Applied Physiotherapy, \\ Polytechnic Institute of Porto, Porto, Health Sciences Institute, Federal University \\ Portugal of Triângulo Mineiro, Uberaba, Brazil \\ ${ }^{3}$ Department of Physiotherapy, Activity and Correspondence \\ Human Movement Study Centre, School of Andreia Noites, School of Allied Health \\ Allied Health Technologies, Polytechnic Technologies, Polytechnic Institute of Porto, \\ Institute of Porto, Porto, Portugal Porto, Portugal. \\ Email: andreianoites@gmail.com
}

\begin{abstract}
Background: Equipment that acts in the reduction in adipose tissue are becoming widely

investigated. One of the resources that begin to gain recognition is radiofre-quency.

Purpose: To verify the existence of scientific evidence and the methodological quality of the articles on the effects of radiofrequency in the reduction in adipose tissue. Methodology: Systematic review with meta-analysis on the effects of radiofre-quency on adipose tissue. The research was carried out using several databases and including experimental studies only in humans. The evaluation of the methodological quality of the articles was done based on the PEDro Scale.

Results: Twelve articles related with adipose tissue and cellulitis were included. There was a great variability of protocols, and the methodological quality was gen-erally low and the methods most used for the evaluation of results were anthro-pometry, photography, and histopathology. Clinical results suggest a positive effect of radiofrequency on the reduction in adipose tissue, proving an increase in adipo-cyte lipolysis. After statistical analysis, it was verified that the anthropometry presented questionable results.

Conclusion: The clinical results of the studies point to the positive effects of radiofrequency on the reduction in adipose tissue; however, the low methodological make this topic still debatable, requiring more controlled studies.
\end{abstract}

KEYWORDS

adipose tissue, cellulitis, lipolysis, radiofrequency

\section{1 | INTRODUCTION}

Ectopic accumulation of adipose tissue or excessive accumulation of adipose tissue in typical or atypical anatomical regions is closely related to an imbalance of the energy balance. This imbalance refers to a higher caloric intake when compared to energy consumption. ${ }^{1}$ This imbalance has cardiovascular and metabolic implications, as well as a negative impact on self-image satisfaction. ${ }^{2,3}$

Radiofrequency (RF) is a form of high-frequency electromagnetic energy that works by heating the tissues. Its action in the deeper tissues (subcutaneous layer) aims to raise cellular metabolism., ${ }^{4,5}$ When applied to tissues, RF generates oscillating magnetic fields that move electrically charged particles, producing heat in the tissues, and the amount of heat produced depends on the resistance (bioimpedance) of the target tissue. ${ }^{6,7}$ Electric energy is converted into thermal 
energy in adipose tissue, which conserves energy because it is not a good conductor of electric current. ${ }^{4}$

There is evidence of a transient RF effect on autonomous homeostasis with no known negative effects. This autonomic response to $\mathrm{RF}$ is reflected in a thermoregulatory vasomotor mechanism, in changes in the activity of chemoreceptors and even in fluctuations of the renin-angiotensin system, responses related to the control of energy metabolism. ${ }^{5}$

The elevation of tissue temperature appears to be sufficient to activate the sympathetic branch of the Autonomic Nervous System, leading to the release of catecholamines (adrenaline and noradrenaline), which are the trigger to activate lipolysis. ${ }^{5,8,9}$

Lipolysis is the reversible biochemical process where the catabolism of the triglycerides, stored in the lipid droplets of the adipocytes, occurs. This process culminates with the generation of nonesterified fatty acids and glycerol. The fatty acids released into the bloodstream can then be used as a substrate to produce energy. 2,10

The available literature on this subject still lacks information that allows us to assume whether the effects of RF are reversible or not and this process can depend on the temperature used and the time of exposure. At the same time, tissue heating may also be related to vasodilation and increased perfusion and local oxygenation that promote oxidation and lipid turnover that will culminate in decreased adipocyte cell volume. ${ }^{11}$ Considering that the processes potentiate each other, the improvement of blood perfusion and the flow of hormones could then make lipolysis more efficient. ${ }^{8}$

Based on the above, the objective of this systematic review with meta-analysis is to verify the existence of scientific evidence and the methodological quality of the articles on the effects of radiofrequency in the reduction in adipose tissue.

\section{2 | METHODS}

The present study, conducted at the School of Allied Health Technologies_Polytechnic Institute of Porto, Portugal, consists of a systematic review of the literature with a meta-analysis based on the Guide to Systematic Review ${ }^{12}$ and performed according to the instructions of the Preferred Reporting Items for Systematic Reviews and Meta-Analysis (PRISMA). ${ }^{13}$

The following databases were consulted: PubMed, Science Direct, Cochrane, and PEDro. Some articles obtained from external sources were also included.

In the research, the following combinations of words were used radiofrequency; TECAR; "Teca Therapy"; "Capacitive Resistive Diathermy Therapy," with the descriptors Lipolysis; "Adipose Tissue"; "Body Fat Distribution"; "Body Mass Index"; "Catecholamines"; "Autonomic Nervous System"; "Sympathetic Nervous System"; "Weight Loss"; "Lipid Mobilization"; Cellulite.

The research was conducted between April 3, 2018 and April 11, 2018 , by three independent reviewers, and in cases of divergence, a consensus among the three reviewers was requested.

The analysis of the scientific and methodological quality of the studies was carried out using the PEDro Scale. ${ }^{14}$ The application of this scale was carried out by two evaluators, independently. In case of discrepancy in the evaluation, it was resolved, primarily through consensus and in case of difficulty, with the participation of a third evaluator.

The authors extracted data on the type of study, characteristics of the participants, type of interventions performed, outcomes evaluated, and the main results obtained for the outcomes of interest previously defined in the present systematic review.

Unpublished studies were not considered, and no contacts were established with the authors of the studies examined.

The meta-analysis was performed for the studies that presented the means and standard deviations of the difference before and after treatment, with $95 \%$ confidence interval, using the software Microsoft Excel and IBM Statistical Package for the Social Sciences (SPSS) 24.

\section{1 | Inclusion criteria}

Studies that met the following criteria were included:

- Articles classified as randomized control trials (RCTs), nonrandomized control trials, clinical trials, case series, and case reports;

- Studies performed only in humans;

- Studies that target adipose tissue or cellulite (on condition that some outcome or mechanism of action related to adipose tissue was explicit in the article);

- Studies published in the English, Portuguese, or Spanish language, without limitation of the publication period

\section{3 | RESULTS}

The PRISMA diagram13 illustrates the research and selection procedures of the studies and how many were considered adequate for this systematic review.

A total of 653 articles were identified in the set of databases used, along with nine articles from external sources. After eliminating duplicate articles, they were subject to title and abstract filtering. At this stage, 65 articles were selected to complete reading. Only 12 of the studies extracted were adequate for a detailed review and considered appropriate for inclusion in this systematic review (Figure 1).

Of the 12 articles selected, nine are classified as pre-experimental and three are RCTs. The studies were published between the years 2006 and 2017.

The mean sample size was $28.12 \pm 12.7$ with the sample being almost exclusively composed of female members in all articles.

The age of the subjects involved in each study ranges from 18 to 82 years. In order to normalize this age range, the mean age limit was calculated (using the values provided by eight of the twelve articles (since four of them concealed this information), and so, the mean of the upper limit age was calculated as being of $61 \pm 8$, 9 years.

All studies present variable methodology; however, evaluations were always performed. Although there were different evaluation periods, there was always a pre- and post-treatment evaluation. There were 
Number of articles indentified in the databases $(n=653)$

(PubMed n=431; Science Direct $n=160$; PEDro $n=0$; Cochrane $n=62$ )

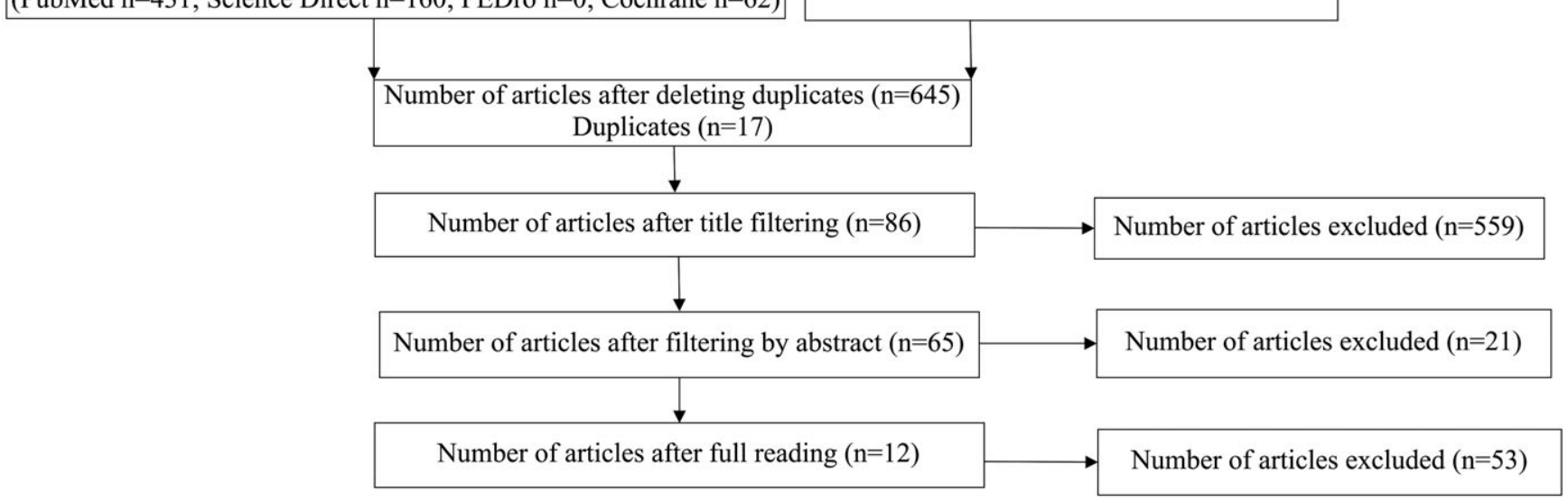

Number of articles from other sources $(n=9)$

FIGURE 1 Sample selection flowchart

10 assessment methods used in the interventions (generally combined), with emphasis on anthropometric measures and photography (Figure 2).

Although all articles refer to temperature, the methods for monitoring it were not generally reported.

The anatomical regions involved were buttocks (three), abdomen (five), and thighs (six), and two studies report intervention on the arms and face.

No references were made to capacitive, resistive, or combined applications. The application mode is not always mentioned, but the multipolar applications (five studies) and unipolar applications (one study) appear to be prominent. The parameter related to the intervention frequencies is also variable $(0.6-27 \mathrm{MHz})$.

The studies seem unanimous about the temperature needed to have an effect on the adipocytes, establishing as ideal a temperature range between 40 and $42^{\circ} \mathrm{C}$ in the epidermis (reaching up to $45^{\circ} \mathrm{C}$, but with a sensation of pain).

Not all studies contained information on how long the temperature was maintained. Pino, et al; Goldberg, Fazeli, and Berlin; Kaplan and Gat; Van Der Lugt, et al; Franco, Kothare, Ronan, Grekin and McCalmont; Levenberg; McDaniel and Samková; Hayre, Palm, and Jenkin refer to the temperature achieved, but only Van Der Lugt, et al, and McDaniel and Samková refer to the minimum maintenance time of the temperature (respectively 12 and 15 minutes). ${ }^{3,4,7,15-19}$

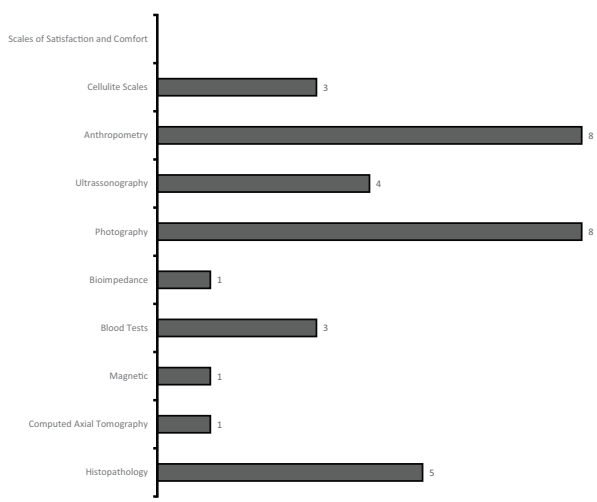

FIGURE 2 Assessment methods used in the studies evaluated
The intensity used also varied between the studies and some did not contain this information. Pino, et al; Goldberg, Fazeli, and Berlin; Van Der Lugt, et al; Levenberg; Mlosek, Wozniak, Malinowska, Lewandowski, and Nowicki; McDaniel and Samková; Suh, et al; Wanitphakdeedecha, Sathaworawong, Manuskiati, and Sadick refer to the intensities used, but these vary with the patient's feedback and with the equipment used. ${ }^{3,4,15,16,18,20-22}$

As for the total number of sessions, they ranged from 1 to 36 , this being an estimate (article refers to home application 2-3 times a week for 3 months). As for the interval between sessions, two articles refer to a 2-week interval and eight articles refer to a 1-week interval. The remaining two articles are not specific to this parameter.

The results of the articles selected for this review are shown in Table 1. The scientific and methodological quality scores of the articles according to the PEDro Scale are shown in Table 2, with one study classified with 2 points, three with 3 points, four with 4 points, two with 5 points and two with 6 points.

The meta-analysis was performed for the studies that presented the means of the differences between the pre- and post-treatment, with their respective standard deviations (five studies) for fourteen variables analyzed. Figure 3 shows the average represented by the point and the size of the interval represented by the traces. The more distant from zero, the greater the improvements obtained. When the range is too large or the lower limit close to zero, it can be considered that the effects are debatable. For the remaining studies, where the measures were presented as a percentage, it was not possible to perform the meta-analysis.

\section{4 | DISCUSSION}

In general, all articles analyzed in the present systematic review demonstrated a clinical improvement, and in some, confirmed by anthropometric and histological studies.

Regarding adverse effects, none of the articles presented serious complications such as burns, scars, or skin irritations. 


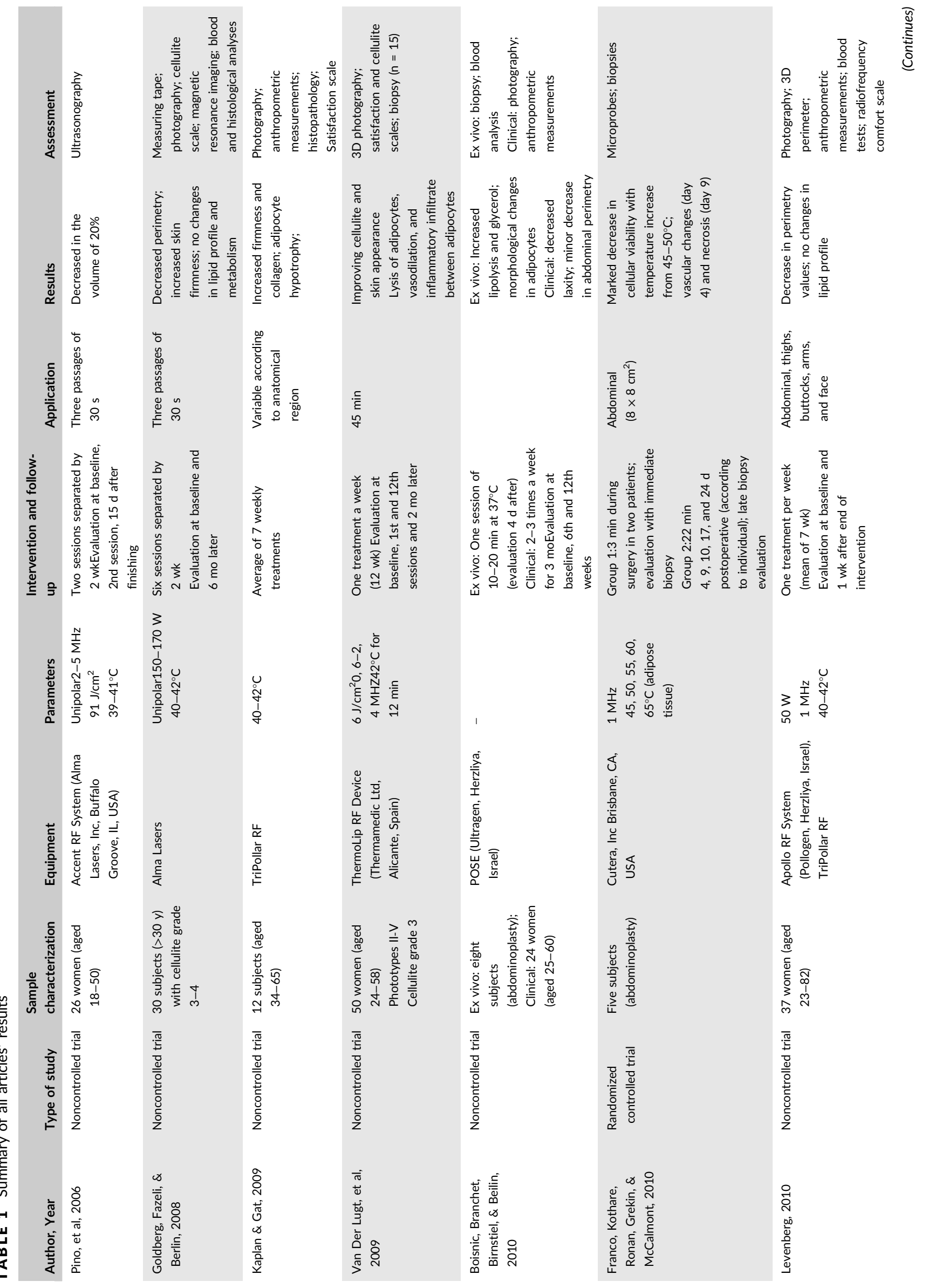




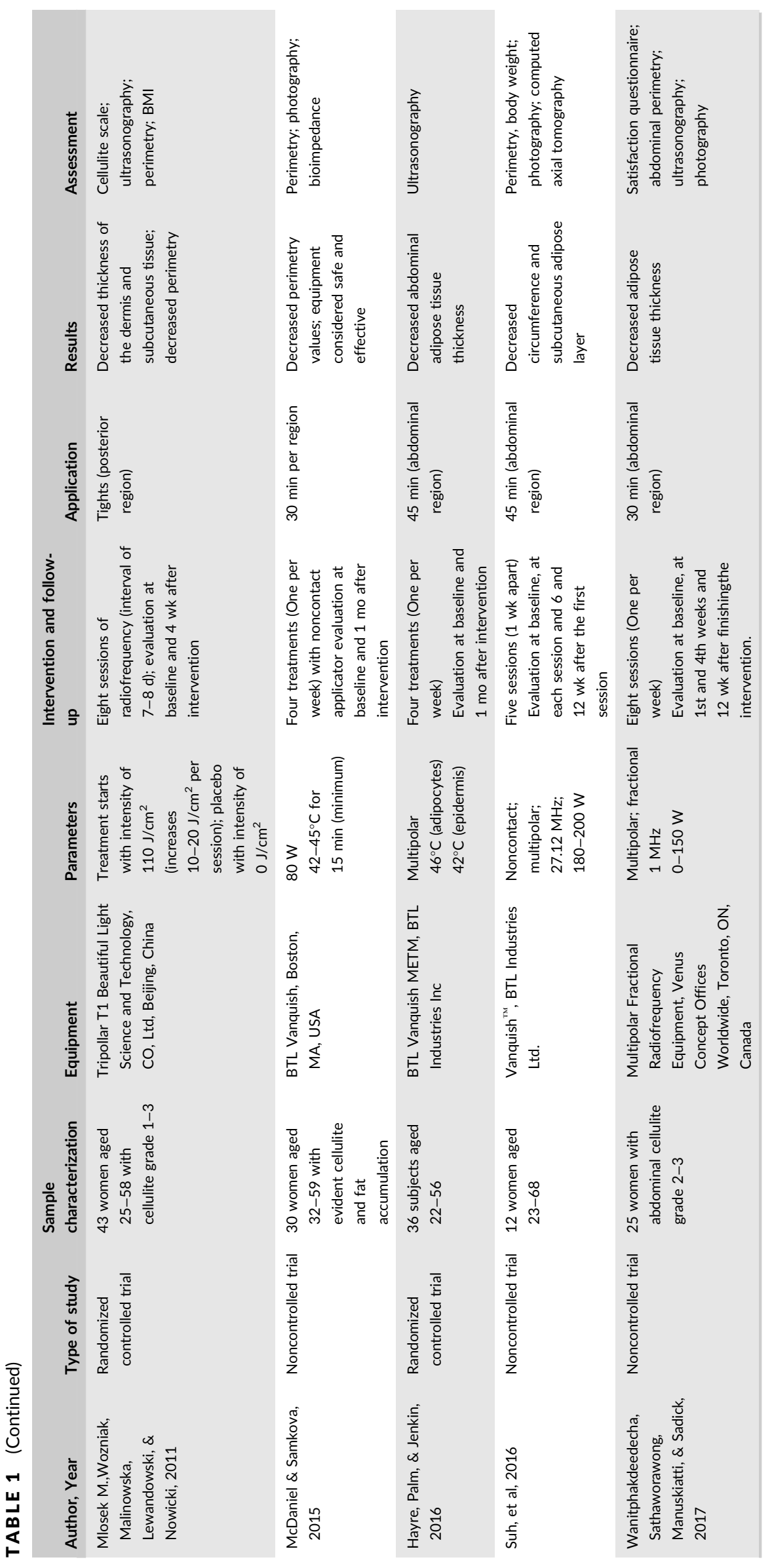




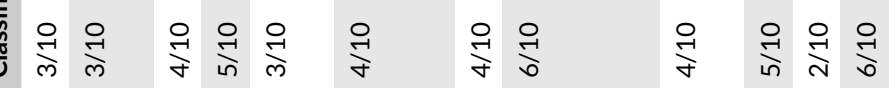

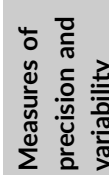

嵒

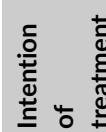

范

要 4 焉

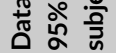

宓

造 0 ०

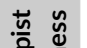

运

㫕

苍館

00000

$\circ$

$\circ 0$

0000

辛

离

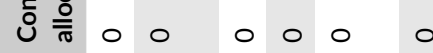

$\circ 00000$

¿

:

$\circ$

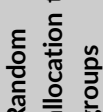

造 产 0

$\circ 000$

$0 \quad 4$

$0 \quad r \quad 0$

琎

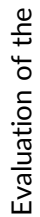

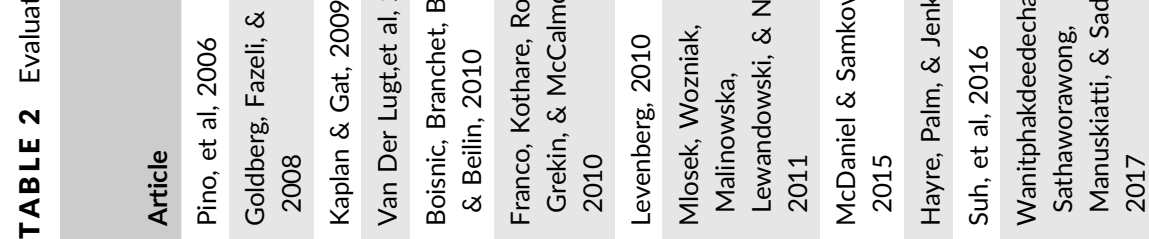




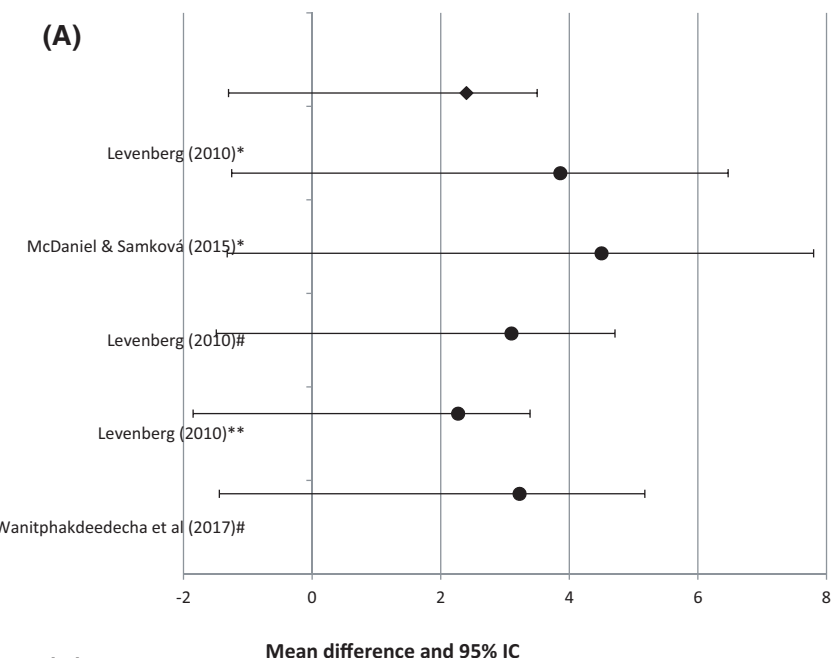

(B)

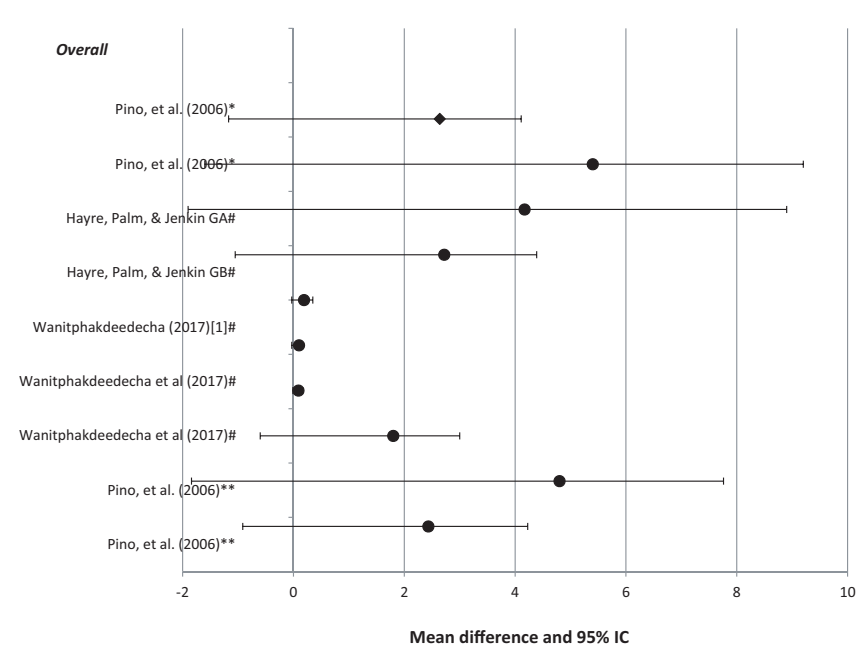

FIGURE 3 Forest plot of means and 95\% IC for perimtry measurements. (A) Perimetry measurements, (B) Ultrassonography measurements

It has been found that there are only a few RCTs and many preexperimental studies, which are not so well listed in the scientific evidence pyramid.

As for the methodological quality of the articles, it can be considered that it is generally low, which makes it difficult to correlate the intervention with the results. The samples used in these articles are usually very small and important variables such as eating habits, physical activity practice, and menstrual cycle (since most of the volunteers were female) are omitted.

The articles present great variability between the protocols, sometimes not specifying them, which make it difficult to standardize and compare the results. Most of the studies present a very wide range of ages that interfere with the analysis and comparison of results due to the inherent alterations of aging.

\subsection{RF in cellulite}

The improvement of the body contour seems to occur mainly due to the diminution of skin laxity. Several authors believe that this is one of the main effects of RF. $4,7,15,16,23$
One explanation that seems to be unanimous among the authors is the thickening and better organization of collagen fibers. ${ }^{4,15,16}$

Another explanation given by most articles is the increase in the amount of collagen. Boisnic, Branchet, Birnstiel, and Beilin and Van Der Lugt et al agree that this increase in collagen is explained by the effects of RF. This triggers an inflammatory reaction in the dermis and protein denaturation. Afterward, by the healing process, there is a significant increase in the activity of the fibroblasts in the dermis, resulting in an increase in collagen synthesis. ${ }^{7,15,18}$

However, justifying the increase in the amount of dermal collagen, Boisnic, Branchet, Birnstiel, and Beilin was the only one that did not find a significant increase in collagen in the histological observations.

The decrease in the perimeter of the RF-treated area may be related to the improvement of laxity since the induced fibrosis causes tissue contraction, thus reducing the distance between the corneal layer and the deep fascia. ${ }^{15,16}$

Suh et al showed a relationship between the reduction in perimetry and the volume of adipose tissue, noting that the decreases in abdominal perimetry values do not directly indicate a decrease in adipose tissue volume or body weight, probably due to the exclusively local effect of RF. Effectively, weight loss does not appear to be a predictable effect of RF. ${ }^{3,22,23}$

\section{2 $\mathrm{RF}$ in the adipose tissue}

Of the five studies that refer specific histological and biochemical analyses for the adipose tissue, some controversy in the results is perceived. Only three of these studies performed blood tests.

Goldberg, Fazeli, and Berlin reported no change in lipid profile. Although the sample has a considerable size and despite referring the use of a unipolar application (15-20 mm depth) with a frequency of $40 \mathrm{~Hz}$ and an intensity between 150 and $170 \mathrm{~W}$ in order to achieve and maintain an epidermal temperature of $40-42^{\circ} \mathrm{C}$, it is not stated for how long that temperature was maintained. ${ }^{16}$

Data from Goldberg, Fazeli, \& Berlin ${ }^{16}$ are in line with the results obtained by Levenberg. ${ }^{18}$ In this study, a tripolar apparatus with a frequency of $1 \mathrm{MHz}$ and a maximum intensity of $50 \mathrm{~W}$ was used. According to the author, RF causes heat that stimulates lipid metabolism. However, the blood test results did not show any acute or late blood changes after RF application.

None of the authors suggests possible explanations for the absence of results at this level; however, Levenberg reports that the heat-induced behavior of tissues is dependent on factors such as maximum temperature reached, exposure time, tissue hydration, and age of the individual. ${ }^{18}$

In contrast, a third study by Boisnic, Branchet, Birnstiel, and Beilin found a significant increase in lipolysis 4 days after a tripolar appliance for home use. In fact, a significant increase in lipolysis $(P=0.04)$ was observed in the RF with an increase in glycerol release, when compared to nontreated fragments. ${ }^{23}$

Regarding the histological analysis of adipose tissue Boisnic, Branchet, Birnstiel, and Beilin observed that in 34\% of adipocytes morphological alterations occurred (such as loss of homogeneity, 
elongation, and irregularities of shape and partial rupture of walls). ${ }^{23}$ This study, despite having a small histological sample, confirms the results already mentioned by Kaplan and Gat. ${ }^{7}$ However, the authors do not present explanations for the results obtained, and since the intervention protocol was not referred, it is not possible to relate the effects with RF parameters.

The data seem to indicate that the RF applied with temperatures within the therapeutic range (up to $42^{\circ} \mathrm{C}$ to the epidermis) seems to fit into the lipolytic intervention, that is, an intervention that leads to adipocyte hypotrophy (by the metabolism of the lipid content) without causing damage in the cell membrane or inflammation. ${ }^{10}$

In this case, the potentiation of lipolysis is justified because the elevation of the temperature stimulates the release of adrenaline which, in turn, potentiates lipolysis. At the same time, the adipose tissue heating is directly related to the vasodilatation phenomenon that generates an increase in perfusion and tissue oxygenation. In this way, oxidation and lipid turnover will be potentiated, culminating in the decrease in adipocyte cell volume (hypotrophy). ${ }^{11}$

Relating the two mechanisms, it is probable that the elevation of the tissue temperature will be enough to activate the sympathetic branch of the Autonomic Nervous System leading to the release of catecholamines (adrenaline and noradrenaline). These are a trigger to activate lipolysis. With such improved blood perfusion, the flow of these hormones is more effective, making lipolysis more efficient. 5,8

Although the temperature facilitates lipolysis, it is necessary to consider the anatomical site of the application of the intervention. Women have greater difficulty in enhancing lipolysis in areas such as thighs, abdomen, and buttocks. This seems to be due to the higher estrogenic production in women, which leads to a higher concentration of alpha-2-adrenergic receptors at these sites, making the lipolytic process difficult. ${ }^{3}$

The maximum temperature reached and the time of exposure seem to be relevant factors for understanding the tissue response to temperature rise. Goldberg, Fazeli, and Berlin report that the protocol used reached temperatures of $40-42{ }^{\circ} \mathrm{C}$ in the epidermis, but does not mention the maintenance time of this temperature. ${ }^{16}$ Levenberg refers to the attainment of a temperature of $40-42^{\circ} \mathrm{C}$ in its protocol. ${ }^{18}$

A study carried out in 2009, Van Der Lugt et al found adipocyte membrane lysis following RF application. The authors used equipment with variable frequency $(0.6-2.4 \mathrm{MHz})$ that was adequate to the temperature of the tissues. This is an advantage since although the tissues have different impedances, it decreases with increasing temperature and, with frequency adjustment, a certain temperature in a target tissue can be kept longer or with greater control (depending on the depth). ${ }^{4}$ Although no late effects were visible, the biopsy performed shortly after the first session showed dilated vessels and cell membrane lysis of the adipocytes with a protocol in which the epidermis was reached $42^{\circ} \mathrm{C}$ at $44^{\circ} \mathrm{C}$ for 12 minutes. The authors justify these results precisely with the temperature reached and with the exposure time. That is, according to Van Der Lugt et al, a temperature to the epidermis of $42-44^{\circ} \mathrm{C}$ equals a temperature of 45 $48^{\circ} \mathrm{C}$ to $20 \mathrm{~mm}$ depth. This difference is due to the low water content of the adipocytes that increase their impedance. The authors report that at these temperatures, protein denaturation occurs and, therefore, membrane lysis of adipocytes. ${ }^{4}$

Although there is a lack of indication of the parameters used, the methodological quality of the studies is not high and the samples are small, there seems to be an indication that RF has an effect on adipose tissue.

Doubt remains as to the reversibility or irreversibility of the effects. It seems, however, that the key may be at the temperature reached and in the time that it is maintained. Franco, Kothare, Ronan, Grekin, and McCalmont report that cell death increases with exposure to temperature (increase in temperature and duration of exposure), noting that a 3-minute cell exposure at a temperature of $45^{\circ} \mathrm{C}$ viability to $40 \% .^{17}$

\section{3 | Limitations}

This review study found several limitations, including the fact that the word "Radiofrequency" is not a valid descriptor. Thus, a large number of articles related to ablative radiofrequency were found which had to be rejected.

On the other hand, the lack of parameters of the protocols used is notorious, preventing the reproduction of the methodologies used.

As for the systematic review itself, it dealt with a small number of articles, not allowing generalizations and making comparisons between the methods and the results obtained.

\section{5 | CONCLUSIONS}

After the review, it is concluded that there is little evidence to prove the effects of RF on adipose tissue and that there is a low methodological quality.

In terms of efficacy, despite the limitations reported in the various studies under review, all considered that RF has a positive effect on adipose tissue and therefore a positive impact on the level of health effects. Therefore, it would be extremely important to carry out studies with a good methodological quality that use metabolic evaluation measures, in order to allow the perception of whether the improvements observed after RF intervention are directly related to adipose tissue. 


\section{REFERENCES}

1. Meyer PF, da Silva RM, Oliveira G et al. Effects of cryolipolysis on abdominal adiposity. Case Rep Dermatol Med. 2016;2016:7.

2. Lass A, Zimmermann R, Oberer M, Zechner R. Lipolysis - a highly regulated multi-enzyme complex mediates the catabolism of cellular fat stores. Prog Lipid Res. 2011;50(1):14-27.

3. McDaniel D, Samkova P. Evaluation of the safety and efficacy of a non-contact radiofrequency device for the improvement in contour and circumferential reduction of the inner and outer thigh. J Drugs Dermatol. 2015;14(12):1422-1424.

4. van der Lugt C, Romero C, Ancona D, Al-Zarouni M, Perera J, Trelles MA. A multicenter study of cellulite treatment with a variable emission radio frequency system. Dermatol Ther. 2009;22(1):74-84.

5. Pumprla J, Howorka K, Kolackova Z, Sovova E. Non-contact radiofrequency-induced reduction of subcutaneous abdominal fat correlates with initial cardiovascular autonomic balance and fat tissue hormones: safety analysis. F1000Res. 2015;4:49.

6. Alexiades-Armenakas M, Dover JS, Arndt KA. Unipolar radiofrequency treatment to improve the appearance of cellulite. J Cosmet Laser Ther. 2008;10(3):148-153.

7. Kaplan H, Gat A. Clinical and histopathological results following TriPollar radiofrequency skin treatments. J Cosmet Laser Ther. 2009;11(2):78-84.

8. Belenky I, Margulis A, Elman M, Bar-Yosef U, Paun SD. Exploring channeling optimized radiofrequency energy: a review of radiofrequency history and applications in esthetic fields. Adv Ther. 2012;29 (3):249-266.

9. Ryden M, Arner P. Subcutaneous Adipocyte lipolysis contributes to circulating lipid levels. Arterioscler Thromb Vasc Biol. 2017;37 (9):1782-1787.

10. Pinto H. Local fat treatments: classification proposal. Adipocyte. 2016;5(1):22-26.

11. Mulholland RS, Paul MD, Chalfoun C. Noninvasive body contouring with radiofrequency, ultrasound, cryolipolysis, and low-level laser therapy. Clin Plast Surg. 2011;38(3):503-520, vii-iii.

12. Gomes IS, CaminhalO. Guia para estudos de revisão sistemática: uma opção metodológica para as ciências do movimento humano. Movimento. 2014;395-411.

13. GalvãoTF, PansaniTD, HarradD. Principais itens para relatar revisões sistemáticas e meta-análises: a recomendação PRISMA. Epidemiol Serv Saúde. 2015;24:335-342.

14. de Morton NA. The PEDro scale is a valid measure of the methodological quality of clinical trials: a demographic study. Aust J Physiother. 2009;55(2):129-133.
15. Emilia del PinoM, RosadoRH, AzuelaA, et al. Effect of controlled volumetric tissue heating with radiofrequency on cellulite and the subcutaneous tissue of the buttocks and thighs. J Drugs Dermatol. 2006;5(8):714-722.

16. Goldberg DJ, Fazeli A, Berlin AL. Clinical, laboratory, and MRI analysis of cellulite treatment with a unipolar radiofrequency device. Dermatol Surg. 2008;34(2):204-209.

17. Franco W, Kothare A, Ronan SJ, Grekin RC, McCalmont TH. Hyperthermic injury to adipocyte cells by selective heating of subcutaneous fat with a novel radiofrequency device: feasibility studies. Lasers Surg Med. 2010;42(5):361-370.

18. Levenberg A. Clinical experience with a TriPollar radiofrequency system for facial and body aesthetic treatments. Eur J Dermatol. 2010;20(5):615-619.

19. Hayre N, Palm M, Jenkin P. A clinical evaluation of a next generation, non-invasive, selective radiofrequency, hands-free, body-shaping device. J Drugs Dermatol. 2016;15(12):1557-1561.

20. Mlosek RK, Woźniak W, Malinowska S, Lewandowski M, Nowicki A. The effectiveness of anticellulite treatment using tripolar radiofrequency monitored by classic and high-frequency ultrasound. J Eur Acad Dermatol Venereol. 2012;26(6):696-703.

21. Suh DH, Kim CM, Lee SJ, Kim H, Yeom SK, Ryu HJ. Safety and efficacy of a non-contact radiofrequency device for body contouring in Asians. J Cosmet Laser Ther. 2017;19(2):89-92.

22. Wanitphakdeedecha R, Sathaworawong A, Manuskiatti W, Sadick NS. Efficacy of multipolar radiofrequency with pulsed magnetic field therapy for the treatment of abdominal cellulite. J Cosmet Laser Ther. 2017;19(4):205-209.

23. Boisnic S, Branchet MC, Birnstiel O, Beilin G. Clinical and histopathological study of the TriPollar home-use device for body treatments. Eur J Dermatol. 2010;20(3):367-372. 